\title{
Special section of business process modeling, development and support (BPMDS) 2019: transformative BPMDS
}

\author{
Jens Gulden ${ }^{1}$
}

Received: 17 September 2021 / Accepted: 20 September 2021 / Published online: 5 October 2021

(c) The Author(s), under exclusive licence to Springer-Verlag GmbH Germany, part of Springer Nature 2021

The business process modeling, development and support (BPMDS) working conference series is a meeting place for researchers and practitioners in the areas of business development and business applications development. By incorporating these views, BPMDS offers a unique community venue that integrates different streams of research on business processes and business information systems, and takes in a broad view on the whole range of BPMDS research and interrelationships among different perspectives. This makes it attractive for authors to publish cutting-edge research results at BPMDS. In this special section, a selection of the most influential contributions to the 2019 edition of the working conference are collected.

\section{Scope}

At the beautiful venue of Rome, the Business Process Modeling, Development, and Support (BPMDS) working conference celebrated its $20^{\text {th }}$ anniversary in 2019. In the light of this event, the leading theme "Transformative BPMDS" was chosen to emphasize a foresighted view on the future of business process modeling and the application of business process models in science and practice.

The working conference received 20 submissions by authors from 10 countries. Nine papers were selected in a blind review process and published in the Springer LNBIP 352 volume. This Software and Systems Modeling special section collects extended versions of 5 selected articles from the conference, which have been intensively revised and reviewed in another two-round blind review process.

$\triangle$ Jens Gulden

jensgulden@acm.org

1 Utrecht University, Utrecht, Netherlands

\section{Selected articles for this special section}

Each of the articles offers a transformation of existing views on business process modeling, by shifting the level of abstraction in modeling, including new modeling perspectives, widening the focus to distributed process execution, or even extending the physical context in which business process models can be applied.

A transformative view on the way how business processes can be modeled is taken in by the article Case model landscapes: toward an improved representation of knowledgeintensive processes using the fCM-language by Fernanda Gonzalez-Lopez, Luise Pufahl, Jorge Munoz-Gama, Valeria Herskovic, and Marcos Sepúlveda. The work introduces an aggregated landscape view on case models for describing knowledge-intensive processes.

The article Language-independent look-ahead for checking multi-perspective declarative process models by Martin Käppel, Lars Ackermann, Stefan Schönig, and Stefan Jablonski makes a contribution to theoretical research on declarative process modeling by suggesting a process simulation approach that incorporates multi-perspective process execution constraints. In line with the first article, this research contributes to strengthening the theoretic knowledge base on non-standard process modeling approaches.

With the contribution of the article Coordinating large distributed relational process structures by Sebastian Steinau, Kevin Andrews, and Manfred Reichert, an architectural perspective is taken in on the nature of processes which are executed in distributed environments. This contributes to transforming the notion of what a process is away from the understanding as an easy to control local phenomenon to a decentral and widely decoupled concourse of activities. The article elaborates fundamental theoretic considerations about the characteristics of such process structures.

In their article Exploring how users engage with hybrid process artifacts based on declarative process models: a behavioral analysis based on eye-tracking and think-aloud, Amine Abbad Andaloussi, Francesca Zerbato, Andrea Burat- 
tin, Tijs Slaats, Thomas T. Hildebrandt, and Barbara Weber perform an empirical analysis of human modeling behavior in environments that allow multiple simultaneous ways to express knowledge about processes. This work unveils complex dependencies between stakeholders, tasks, and modeling techniques, which shows that transformations in the modeling environment and combinations of different modeling techniques are a rich research field which holds out the prospect of further fruitful insights.

An exciting approach for transforming where and how process modeling is performed is developed in the article HoloFlows: modelling of processes for the Internet of Things in mixed reality by Ronny Seiger, Romina Kühn, Mandy Korzetz, and Uwe Aßmann. The presented approach takes process modeling to the workplace by allowing to mix real-world actions with virtual reality components in which modeling is performed. Contributions like this convincingly show that the field of BPMDS remains in an ongoing flow of development and that there are many new directions into which the field can and will develop in the future.

Back when BPMDS 2019 was held, no one could possibly anticipate how transformative the upcoming times would become. Months later the world began to face the global Covid-19 pandemic. Our lives have been disrupted in many ways since then, with impactful influences on how we work, how we live in our homes, and how we experience culture and society. BPMDS is embedded into this world of ongoing transformations. As a consequence, our research field faces strong challenges regarding the economic, societal, and ethical implications it is based on. Traditional perspectives on BPMDS often regarded business process modeling as a tool to increase efficiency and control over processes. While these remain relevant, in times of disruption we have learned that in order to better understand the nature of business processes, more than control and operational consistency are relevant. Motivational influences of stakeholders, resonances of processes with their socio-economical contexts, and externalities that burden the environment of processes, demand for new systematic methodical means to be incorporated in future business process modeling languages and their applications.
Our discipline no longer only is faced with questions about what we can do with business process modeling, development and support of process-oriented information systems. We should increasingly address ethical questions on whether and how we should do it. For research on business processes, business process management, and BPMDS, this means a plethora of innovative developments expected to be lying ahead.

Acknowledgements I wish to thank all authors from the 2019 edition of BPMDS for their high-quality contributions to the conference and for creating extended revisions for this special section. I also would like to express my gratitude to the members of the BPMDS program committee and all reviewers, without whose careful assessments and critical suggestions the articles would not have reached the degree of quality they have. Last but not least, my thanks go to the editors-in-chief of the Journal of Software and Systems Modeling, especially Martin Schindler for his support.

Publisher's Note Springer Nature remains neutral with regard to jurisdictional claims in published maps and institutional affiliations.

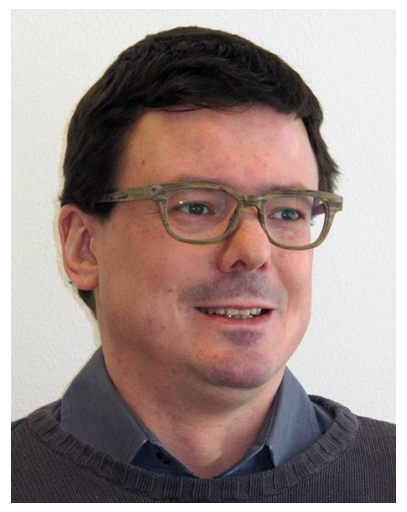

Jens Gulden is a member of the Business Process Management \& Analytics group at Utrecht University. He has a Ph.D. in Business Informatics and a master degree in Philosophy. In his research he examines and develops methods for designing visual modeling languages and knowledge visualizations. He also works on ethical analysis and elicitation of ethical conflicts in the field of business informatics. His teaching activities cover responsible use of information technology, advanced research methods, architectures of digital ecosystems, and responsible data science. Jens Gulden has been a co-organizer of the BPMDS working conference at CAiSE and was in charge of the ModTools workshop on methodical development of modeling tools. He annually organizes the EMoBI workshop on ethics and morality in business informatics. 